IJMMS 2003:9, 539-547

PII. S0161171203202027

http://ijmms.hindawi.com

(c) Hindawi Publishing Corp.

\title{
A BASIC INEQUALITY FOR SUBMANIFOLDS IN A COSYMPLECTIC SPACE FORM
}

\author{
JEONG-SIK KIM and JAEDONG CHOI
}

\author{
Received 7 February 2002
}

\begin{abstract}
For submanifolds tangent to the structure vector field in cosymplectic space forms, we establish a basic inequality between the main intrinsic invariants of the submanifold, namely, its sectional curvature and scalar curvature on one side; and its main extrinsic invariant, namely, squared mean curvature on the other side. Some applications, including inequalities between the intrinsic invariant $\delta_{M}$ and the squared mean curvature, are given. The equality cases are also discussed.
\end{abstract}

2000 Mathematics Subject Classification: 53C40, 53D15.

1. Introduction. To find simple relationships between the main extrinsic invariants and the main intrinsic invariants of a submanifold is one of the natural interests of the submanifold theory. Let $M$ be an $n$-dimensional Riemannian manifold. For each point $p \in M$, let $(\inf K)(p)=\inf \{K(\pi)$ : plane sections $\pi \subset$ $\left.T_{p} M\right\}$. Then, the well-defined intrinsic invariant $\delta_{M}$ of $M$ introduced by Chen [4] is

$$
\delta_{M}(p)=\tau(p)-(\inf K)(p),
$$

where $\tau$ is the scalar curvature of $M$ (see also [6]).

In [3], Chen established the following basic inequality involving the intrinsic invariant $\delta_{M}$ and the squared mean curvature for $n$-dimensional submanifolds $M$ in a real space form $R(c)$ of constant sectional curvature $c$ :

$$
\delta_{M} \leq \frac{n^{2}(n-2)}{2(n-1)}\|H\|^{2}+\frac{1}{2}(n+1)(n-2) c .
$$

The above inequality is also true for anti-invariant submanifolds in complex space forms $\widetilde{M}(4 c)$ as remarked in [7]. In [5], he proved a general inequality for an arbitrary submanifold of a dimension greater than 2 in a complex space form. Applying this inequality, he showed that (1.2) is also valid for arbitrary submanifolds in the complex hyperbolic space $\mathrm{CH}^{m}(4 c)$. He also established the basic inequality for a submanifold in a complex projective space $\mathrm{CP}^{m}$.

A submanifold normal to the structure vector field $\xi$ of a contact manifold is anti-invariant. Thus, the $C$-totally real submanifolds in a Sasakian manifold are anti-invariant as they are normal to $\xi$. An inequality similar to (1.2) for $C$-totally 
real submanifolds in a Sasakian space form $\tilde{M}(c)$ of constant $\varphi$-sectional curvature $c$ is given in [8]. In [9], for submanifolds in a Sasakian space form $\tilde{M}(c)$ tangential to the structure vector field $\xi$, a basic inequality, along with some applications, is presented.

There is another interesting class of almost contact metric manifolds, namely, cosymplectic manifolds [10]. In this paper, submanifolds tangent to the structure vector field $\xi$ in cosymplectic space forms are studied. Section 2 contains the necessary details of submanifolds and cosymplectic space forms for further use. In Section 3, for submanifolds tangent to the structure vector field $\xi$ in cosymplectic space forms, we establish a basic inequality between the main intrinsic invariants, namely, its sectional curvature function $K$ and its scalar curvature function $\tau$ of the submanifold on the one side, and its main extrinsic invariant, namely, its mean curvature function $\|H\|$ on the other side. In Section 4 , we give some applications including inequalities between the intrinsic invariant $\delta_{M}$ and the extrinsic invariant $\|H\|$. We also discuss the equality cases.

2. Preliminaries. Let $\tilde{M}$ be a $(2 m+1)$-dimensional almost contact manifold [2] endowed with an almost contact structure $(\varphi, \xi, \eta)$, that is, $\varphi$ is a $(1,1)$ tensor field, $\xi$ is a vector field, and $\eta$ is 1 -form such that

$$
\varphi^{2}=-I+\eta \otimes \xi, \quad \eta(\xi)=1 .
$$

Then, $\varphi(\xi)=0$ and $\eta \circ \varphi=0$.

Let $g$ be a compatible Riemannian metric with $(\varphi, \xi, \eta)$, that is, $g(\varphi X, \varphi Y)=$ $g(X, Y)-\eta(X) \eta(Y)$ or, equivalently, $g(X, \varphi Y)=-g(\varphi X, Y)$ and $g(X, \xi)=$ $\eta(X)$ for all $X, Y \in T \tilde{M}$. Then, $\tilde{M}$ becomes an almost contact metric manifold equipped with an almost contact metric structure $(\varphi, \xi, \eta, g)$. An almost contact metric manifold is cosymplectic [2] if $\tilde{\nabla}_{X} \varphi=0$, where $\tilde{\nabla}$ is the Levi-Civita connection of the Riemannian metric $g$. From the formula $\tilde{\nabla}_{X} \varphi=0$, it follows that $\tilde{\nabla}_{X} \xi=0$.

A plane section $\sigma$ in $T_{p} \tilde{M}$ of an almost contact metric manifold $\tilde{M}$ is called a $\varphi$-section if $\sigma \perp \xi$ and $\varphi(\sigma)=\sigma$. The $(2 m+1)$-dimensional almost contact manifold $\tilde{M}$ is of the constant $\varphi$-sectional curvature if the sectional curvature $\tilde{K}(\sigma)$ does not depend on the choice of the $\varphi$-section $\sigma$ of $T_{p} \tilde{M}$ and the choice of a point $p \in \tilde{M}$. A cosymplectic manifold $\tilde{M}$ is of the constant $\varphi$-sectional curvature $c$ if and only if its curvature tensor $\tilde{R}$ is of the form [10]

$$
\begin{aligned}
4 \tilde{R}(X, Y, Z, W)=c\{ & g(X, W) g(Y, Z)-g(X, Z) g(Y, W) \\
& +g(X, \varphi W) g(Y, \varphi Z)-g(X, \varphi Z) g(Y, \varphi W) \\
& -2 g(X, \varphi Y) g(Z, \varphi W) \\
& -g(X, W) \eta(Y) \eta(Z)+g(X, Z) \eta(Y) \eta(W) \\
& -g(Y, Z) \eta(X) \eta(W)+g(Y, W) \eta(X) \eta(Z)\} .
\end{aligned}
$$


Let $M$ be an $(n+1)$-dimensional submanifold of a manifold $\tilde{M}$ equipped with a Riemannian metric $g$. The Gauss and Weingarten formulae are given, respectively, by

$$
\tilde{\nabla}_{X} Y=\nabla_{X} Y+h(X, Y), \quad \tilde{\nabla}_{X} N=-A_{N} X+\nabla_{X}^{\perp} N
$$

for all $X, Y \in T M$ and $N \in T^{\perp} M$, where $\tilde{\nabla}, \nabla$, and $\nabla^{\perp}$, respectively, are the Riemannian, induced Riemannian, and induced normal connections in $\tilde{M}, M$, and the normal bundle $T^{\perp} M$ of $M$, respectively, and $h$ is the second fundamental form related to the shape operator $A$ by $g(h(X, Y), N)=g\left(A_{N} X, Y\right)$.

Let $\left\{e_{1}, \ldots, e_{n+1}\right\}$ be an orthonormal basis of the tangent space $T_{p} M$. The mean curvature vector $H(p)$ at $p \in M$ is

$$
H(p)=\frac{1}{n+1} \sum_{i=1}^{n+1} h\left(e_{i}, e_{i}\right)
$$

The submanifold $M$ is totally geodesic in $\tilde{M}$ if $h=0$ and minimal if $H=0$. We put

$$
h_{i j}^{r}=g\left(h\left(e_{i}, e_{j}\right), e_{r}\right), \quad\|h\|^{2}=\sum_{i, j=1}^{n+1} g\left(h\left(e_{i}, e_{j}\right), h\left(e_{i}, e_{j}\right)\right),
$$

where $\left\{e_{n+2}, \ldots, e_{2 m+1}\right\}$ is an orthonormal basis of $T_{p}^{\perp} M$ and $r=n+2, \ldots$, $2 m+1$.

3. A basic inequality. Let $M$ be a submanifold of an almost contact metric manifold. For $X \in T M$, let

$$
\varphi X=P X+F X, \quad P X \in T M, F X \in T^{\perp} M .
$$

Thus, $P$ is an endomorphism of the tangent bundle of $M$ and satisfies

$$
g(X, P Y)=-g(P X, Y), \quad X, Y \in T M
$$

For a plane section $\pi \subset T_{p} M$ at a point $p \in M$,

$$
\alpha(\pi)=g\left(e_{1}, P e_{2}\right)^{2}, \quad \beta(\pi)=\left(\eta\left(e_{1}\right)\right)^{2}+\left(\eta\left(e_{2}\right)\right)^{2}
$$

are real numbers in the closed unit interval $[0,1]$, which are independent of the choice of the orthonormal basis $\left\{e_{1}, e_{2}\right\}$ of $\pi$.

We recall the following lemma from [3]. 
LEMMA 3.1. If $a_{1}, \ldots, a_{n+1}, a$ are $n+2(n \geq 1)$ real numbers such that

$$
\left(\sum_{i=1}^{n+1} a_{i}\right)^{2}=n\left(\sum_{i=1}^{n+1} a_{i}^{2}+a\right)
$$

then $2 a_{1} a_{2} \geq a$, with equality holding if and only if $a_{1}+a_{2}=a_{3}=\cdots=a_{n+1}$.

Now, we prove the following theorem.

THEOREM 3.2. Let $M$ be an $(n+1)$-dimensional $(n \geq 2)$ submanifold isometrically immersed in a $(2 m+1)$-dimensional cosymplectic space form $\tilde{M}(c)$ such that the structure vector field $\xi$ is tangent to $M$. Then, for each point $p \in M$ and each plane section $\pi \subset T_{p} M$, we have

$$
\begin{aligned}
\tau-K(\pi) \leq & \frac{(n+1)^{2}(n-1)}{2 n}\|H\|^{2} \\
& +\frac{c}{8}\left(3\|P\|^{2}-6 \alpha(\pi)+2 \beta(\pi)+(n+1)(n-2)\right) .
\end{aligned}
$$

The equality in (3.5) holds at $p \in M$ if and only if there exists an orthonormal basis $\left\{e_{1}, \ldots, e_{n+1}\right\}$ of $T_{p} M$ and an orthonormal basis $\left\{e_{n+2}, \ldots, e_{2 m+1}\right\}$ of $T_{p}^{\perp} M$ such that

(a) $\pi=\operatorname{Span}\left\{e_{1}, e_{2}\right\}$,

(b) the forms of the shape operators $A_{r} \equiv A_{e_{r}}, r=n+2, \ldots, 2 m+1$, become

$$
\begin{aligned}
A_{n+2} & =\left(\begin{array}{ccc}
\lambda & 0 & 0 \\
0 & \mu & 0 \\
0 & 0 & (\lambda+\mu) I_{n-1}
\end{array}\right), \\
A_{r} & =\left(\begin{array}{ccc}
h_{11}^{r} & h_{12}^{r} & 0 \\
h_{12}^{r} & -h_{11}^{r} & 0 \\
0 & 0 & 0_{n-1}
\end{array}\right), \quad r=n+3, \ldots, 2 m+1 .
\end{aligned}
$$

Proof. In view of the Gauss equation and (2.2), the scalar curvature and the mean curvature of $M$ are related by

$$
2 \tau=\frac{c}{4}\left(3\|P\|^{2}+n(n-1)\right)+(n+1)^{2}\|H\|^{2}-\|h\|^{2},
$$

where $\|P\|^{2}$ is given by

$$
\|P\|^{2}=\sum_{i, j=1}^{n+1} g\left(e_{i}, P e_{j}\right)^{2}
$$


for any local orthonormal basis $\left\{e_{1}, e_{2}, \ldots, e_{n+1}\right\}$ for $T_{p} M$. We introduce

$$
\rho=2 \tau-\frac{(n+1)^{2}(n-1)}{n}\|H\|^{2}-\frac{c}{4}\left(3\|P\|^{2}+n(n-1)\right) .
$$

From (3.7) and (3.9), we get

$$
(n+1)^{2}\|H\|^{2}=n\left(\|h\|^{2}+\rho\right) .
$$

Let $p$ be a point of $M$ and let $\pi \subset T_{p} M$ be a plane section at $p$. We choose an orthonormal basis $\left\{e_{1}, e_{2}, \ldots, e_{n+1}\right\}$ for $T_{p} M$ and $\left\{e_{n+2}, \ldots, e_{2 m+1}\right\}$ for the normal space $T_{p}^{\perp} M$ at $p$ such that $\pi=\operatorname{Span}\left\{e_{1}, e_{2}\right\}$ and the mean curvature vector $H(p)$ is parallel to $e_{n+2}$; then from (3.10), we get

$$
\left(\sum_{i=1}^{n+1} h_{i i}^{n+2}\right)^{2}=n\left(\sum_{i=1}^{n+1}\left(h_{i i}^{n+2}\right)^{2}+\sum_{i \neq j}\left(h_{i j}^{n+2}\right)^{2}+\sum_{r=n+3}^{2 m+1} \sum_{i, j=1}^{n+1}\left(h_{i j}^{r}\right)^{2}+\rho\right) .
$$

Using Lemma 3.1, from (3.11) we obtain

$$
h_{11}^{n+2} h_{22}^{n+2} \geq \frac{1}{2}\left\{\sum_{i \neq j}\left(h_{i j}^{n+2}\right)^{2}+\sum_{r=n+3}^{2 m+1} \sum_{i, j=1}^{n+1}\left(h_{i j}^{r}\right)^{2}+\rho\right\} .
$$

From the Gauss equation and (2.2), we also have

$$
K(\pi)=\frac{c}{4}(1+3 \alpha(\pi)-\beta(\pi))+h_{11}^{n+2} h_{22}^{n+2}-\left(h_{12}^{n+2}\right)^{2}+\sum_{r=n+3}^{2 m+1}\left(h_{11}^{r} h_{22}^{r}-\left(h_{12}^{r}\right)^{2}\right) .
$$

Thus, we have

$$
\begin{aligned}
K(\pi) \geq & \frac{c}{4}(1+3 \alpha(\pi)-\beta(\pi))+\frac{1}{2} \rho+\sum_{r=n+2}^{2 m+1} \sum_{j>2}\left\{\left(h_{1 j}^{r}\right)^{2}+\left(h_{2 j}^{r}\right)^{2}\right\} \\
& +\frac{1}{2} \sum_{i \neq j>2}\left(h_{i j}^{n+2}\right)^{2}+\frac{1}{2} \sum_{r=n+3}^{2 m+1} \sum_{i, j>2}\left(h_{i j}^{r}\right)^{2}+\frac{1}{2} \sum_{r=n+3}^{2 m+1}\left(h_{11}^{r}+h_{22}^{r}\right)^{2},
\end{aligned}
$$

or

$$
K(\pi) \geq \frac{c}{4}(1+3 \alpha(\pi)-\beta(\pi))+\frac{1}{2} \rho,
$$

which, in view of (3.9), yields (3.5). 
If the equality in (3.5) holds, then the inequalities given by (3.12) and (3.14) become equalities. In this case, we have

$$
\begin{gathered}
h_{1 j}^{n+2}=0, \quad h_{2 j}^{n+2}=0, \quad h_{i j}^{n+2}=0, \quad i \neq j>2 ; \\
h_{1 j}^{r}=h_{2 j}^{r}=h_{i j}^{r}=0, \quad r=n+3, \ldots, 2 m+1 ; i, j=3, \ldots, n+1 ; \\
h_{11}^{n+3}+h_{22}^{n+3}=\cdots=h_{11}^{2 m+1}+h_{22}^{2 m+1}=0 .
\end{gathered}
$$

Furthermore, we may choose $e_{1}$ and $e_{2}$ so that $h_{12}^{n+2}=0$. Moreover, by applying Lemma 3.1, we also have

$$
h_{11}^{n+2}+h_{22}^{n+2}=h_{33}^{n+2}=\cdots=h_{n+1 n+1}^{n+2} .
$$

Thus, choosing a suitable orthonormal basis $\left\{e_{1}, \ldots, e_{2 m+1}\right\}$, the shape operator of $M$ becomes of the form given by (3.6). The converse is straightforward.

4. Some applications. For the case $c=0$, from (3.5) we have the following pinching result.

Proposition 4.1. Let $M$ be an $(n+1)$-dimensional $(n>1)$ submanifold isometrically immersed in a $(2 m+1)$-dimensional cosymplectic space form $\tilde{M}(c)$ with $c=0$ such that $\xi \in T M$. Then,

$$
\delta_{M} \leq \frac{(n+1)^{2}(n-1)}{2 n}\|H\|^{2} .
$$

A submanifold $M$ of an almost contact metric manifold $\tilde{M}$ with $\xi \in T M$ is called a semi-invariant submanifold [1] of $\tilde{M}$ if $T M=\mathscr{D} \oplus \mathscr{D}^{\perp} \oplus\{\xi\}$, where $\mathscr{D}=T M \cap \varphi(T M)$ and $\mathscr{D}^{\perp}=T M \cap \varphi\left(T^{\perp} M\right)$. In fact, the condition $T M=\mathscr{D} \oplus$ $\mathscr{D}^{\perp} \oplus\{\xi\}$ implies that the endomorphism $P$ is an $f$-structure [12] on $M$ with $\operatorname{rank}(P)=\operatorname{dim}(\mathscr{D})$. A semi-invariant submanifold of an almost contact metric manifold becomes an invariant or anti-invariant submanifold according as the anti-invariant distribution $\mathscr{D}^{\perp}$ is $\{0\}$ or the invariant distribution $\mathscr{D}$ is $\{0\}[1,12]$.

Now, we establish two inequalities in the following theorems, which are analogous to that of (1.2).

THEOREM 4.2. Let $M$ be an $(n+1)$-dimensional $(n>1)$ submanifold isometrically immersed in a $(2 m+1)$-dimensional cosymplectic space form $\tilde{M}(c)$ such that the structure vector field $\xi$ is tangent to $M$. If $c<0$, then

$$
\delta_{M} \leq \frac{(n+1)^{2}(n-1)}{2 n}\|H\|^{2}+\frac{1}{2}(n+1)(n-2) \frac{c}{4} .
$$


The equality in (4.2) holds if and only if $M$ is a semi-invariant submanifold with $\operatorname{dim}(\mathscr{D})=2$.

Proof. Since $c<0$, in order to estimate $\delta_{M}$, we minimize $3\|P\|^{2}-6 \alpha(\pi)+$ $2 \beta(\pi)$ in (3.5). For an orthonormal basis $\left\{e_{1}, \ldots, e_{n+1}\right\}$ of $T_{p} M$ with $\pi=\operatorname{span}\left\{e_{1}\right.$, $\left.e_{2}\right\}$, we write

$$
\|P\|^{2}-2 \alpha(\pi)=\sum_{i, j=3}^{n+1} g\left(e_{i}, \varphi e_{j}\right)^{2}+2 \sum_{j=3}^{n+1}\left\{g\left(e_{1}, \varphi e_{j}\right)^{2}+g\left(e_{2}, \varphi e_{j}\right)^{2}\right\} .
$$

Thus, we see that the minimum value of $3\|P\|^{2}-6 \alpha(\pi)+2 \beta(\pi)$ is zero provided $\pi=\operatorname{span}\left\{e_{1}, e_{2}\right\}$ is orthogonal to $\xi$ and $\operatorname{span}\left\{\varphi e_{j} \mid j=3, \ldots, n\right\}$ is orthogonal to the tangent space $T_{p} M$. Thus, we have (4.2) with equality case holding if and only if $M$ is semi-invariant such that $\operatorname{dim}(\mathscr{D})=2$ with $\beta=0$.

THEOREM 4.3. Let $M$ be an $(n+1)$-dimensional $(n>1)$ submanifold isometrically immersed in a $(2 m+1)$-dimensional cosymplectic space form $\tilde{M}(c)$ such that $\xi \in T M$. If $c>0$, then

$$
\delta_{M} \leq \frac{(n+1)^{2}(n-1)}{2 n}\|H\|^{2}+\frac{1}{2} n(n+2) \frac{c}{4} .
$$

The equality in (4.4) holds if and only if $M$ is an invariant submanifold.

Proof. Since $c>0$, in order to estimate $\delta_{M}$, we maximize $3\|P\|^{2}-6 \alpha(\pi)+$ $2 \beta(\pi)$ in (3.5). We observe that the maximum of $3\|P\|^{2}-6 \alpha(\pi)+2 \beta(\pi)$ is attained for $\|P\|^{2}=n, \alpha(\pi)=0$, and $\beta(\pi)=1$, that is, $M$ is an invariant and $\xi \in \pi$. Thus, we obtain (4.4) with equality case if and only if $M$ is invariant with $\beta=1$.

In last, we prove the following theorem.

THEOREM 4.4. If $M$ is an $(n+1)$-dimensional $(n>1)$ submanifold isometrically immersed in a $(2 m+1)$-dimensional cosymplectic space form $\tilde{M}(c)$ such that $c>0, \xi \in T M$ and

$$
\delta_{M}=\frac{(n+1)^{2}(n-1)}{2 n}\|H\|^{2}+\frac{1}{2} n(n+2) \frac{c}{4},
$$

then $M$ is a totally geodesic cosymplectic space form $M(c)$.

Proof. In view of Theorem 4.3, $M$ is an odd-dimensional invariant submanifold of the cosymplectic space form $\tilde{M}(c)$. For every point $p \in M$, we can choose an orthonormal basis $\left\{e_{1}=\xi, e_{2}, \ldots, e_{n+1}\right\}$ for $T_{p} M$ and $\left\{e_{n+2}, \ldots, e_{2 m+1}\right\}$ for $T_{p}^{\perp} M$ such that $A_{r}(r=n+2, \ldots, 2 m+1)$ take the form (3.6). Since $M$ is an 
invariant submanifold of a cosymplectic manifold, therefore, it is minimal and $A_{r} \varphi+\varphi A_{r}=0, r=n+2, \ldots, 2 m+1$ [11]. Thus, all the shape operators take the form

$$
A_{r}=\left(\begin{array}{ccc}
c_{r} & d_{r} & 0 \\
d_{r} & -c_{r} & 0 \\
0 & 0 & 0_{n-1}
\end{array}\right), \quad r=n+2, \ldots, 2 m+1
$$

Since $A_{r} \varphi e_{1}=0, r=n+2, \ldots, 2 m+1$, from $A_{r} \varphi+\varphi A_{r}=0$, we get $\varphi A_{r} e_{1}=0$. Applying $\varphi$ to this equation, we obtain $A_{r} e_{1}=\eta\left(A_{r} e_{1}\right) \xi=\eta\left(A_{r} e_{1}\right) e_{1}$; thus, $d_{r}=0, r=n+2, \ldots, 2 m+1$. This implies that $A_{r} e_{2}=-c_{r} e_{2}$. Applying $\varphi$ to both sides, in view of $A_{r} \varphi+\varphi A_{r}=0$ we get $A_{r} \varphi e_{2}=c_{r} \varphi e_{2}$. Since $\varphi e_{2}$ is orthogonal to $\xi$ and $e_{2}$ and $\varphi$ has a maximal rank, the principal curvature $c_{r}$ is zero. Hence, $M$ becomes totally geodesic. As in [12, Proposition 1.3, page 313], it is easy to show that $M$ is a cosymplectic manifold of the constant $\varphi$-sectional curvature $c$.

ACKNOWLEDGMENT. This work was supported by the Korea Science \& Engineering Foundation grant R01-2001-00003.

\section{REFERENCES}

[1] A. Bejancu, Geometry of CR-Submanifolds, Mathematics and Its Applications (East European Series), vol. 23, D. Reidel Publishing, Dordrecht, 1986.

[2] D. E. Blair, Contact Manifolds in Riemannian Geometry, Lecture Notes in Mathematics, vol. 509, Springer-Verlag, Berlin, 1976.

[3] B.-Y. Chen, Some pinching and classification theorems for minimal submanifolds, Arch. Math. (Basel) 60 (1993), no. 6, 568-578.

[4] _ A Riemannian invariant for submanifolds in space forms and its applications, Geometry and Topology of Submanifolds, VI (Leuven, 1993/Brussels, 1993), World Scientific Publishing, New Jersey, 1994, pp. 58-81.

[5] _ A general inequality for submanifolds in complex-space-forms and its applications, Arch. Math. (Basel) 67 (1996), no. 6, 519-528.

[6] _ _ Riemannian submanifolds, Handbook of Differential Geometry, Vol. I (F. Dillen and L. Verstraelen, eds.), North-Holland Publishing, Amsterdam, 2000, pp. 187-418.

[7] B.-Y. Chen, F. Dillen, L. Verstraelen, and L. Vrancken, An exotic totally real minimal immersion of $S^{3}$ in $\mathrm{CP}^{3}$ and its characterisation, Proc. Roy. Soc. Edinburgh Sect. A 126 (1996), no. 1, 153-165.

[8] F. Defever, I. Mihai, and L. Verstraelen, B.-Y. Chen's inequality for C-totally real submanifolds of Sasakian space forms, Boll. Un. Mat. Ital. B (7) 11 (1997), no. 2, 365-374.

[9] Y. H. Kim and D.-S. Kim, A basic inequality for submanifolds in Sasakian space forms, Houston J. Math. 25 (1999), no. 2, 247-257.

[10] G. D. Ludden, Submanifolds of cosymplectic manifolds, J. Differential Geometry 4 (1970), 237-244.

[11] M. M. Tripathi, Almost semi-invariant submanifolds of trans-Sasakian manifolds, J. Indian Math. Soc. (N.S.) 62 (1996), no. 1-4, 225-245. 
[12] K. Yano and M. Kon, Structures on Manifolds, Series in Pure Mathematics, vol. 3, World Scientific Publishing, Singapore, 1984.

Jeong-Sik Kim: Department of Mathematics Education, Sunchon National University, Sunchon 540-742, Korea

E-mail address: jskim01@hanmi r.com

Jaedong Choi: Department of Mathematics, P.O. Box 335-2, Airforce Academy, Ssangsu, Namil, Chungwon, Chungbuk 363-849, Korea

E-mail address: jdong@afa. ac. kr 


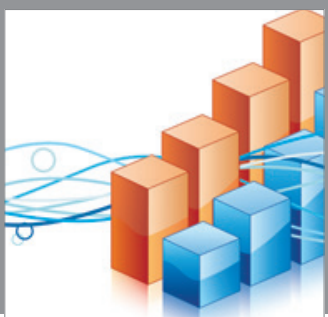

Advances in

Operations Research

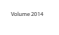

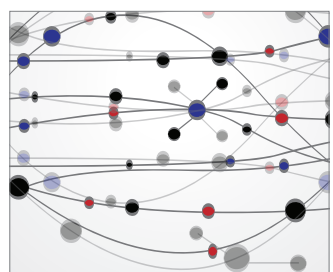

\section{The Scientific} World Journal
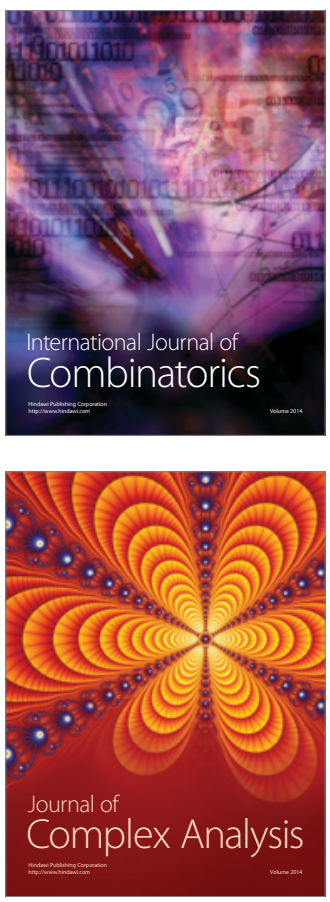

International Journal of

Mathematics and

Mathematical

Sciences
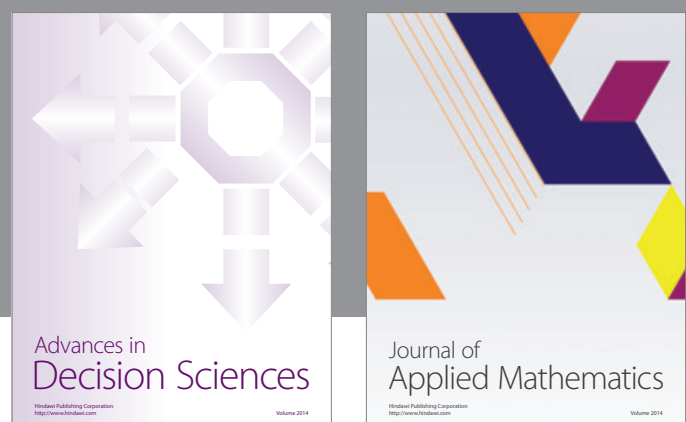

Journal of

Applied Mathematics
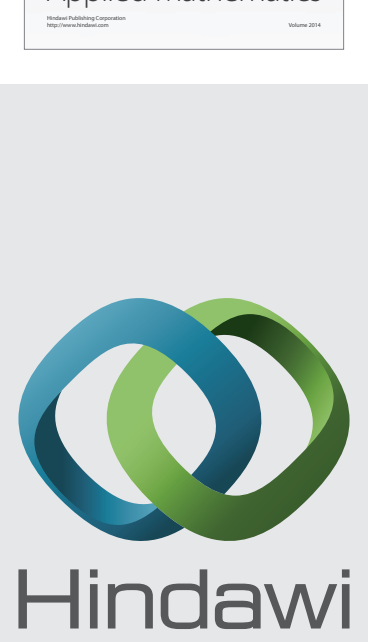

Submit your manuscripts at http://www.hindawi.com
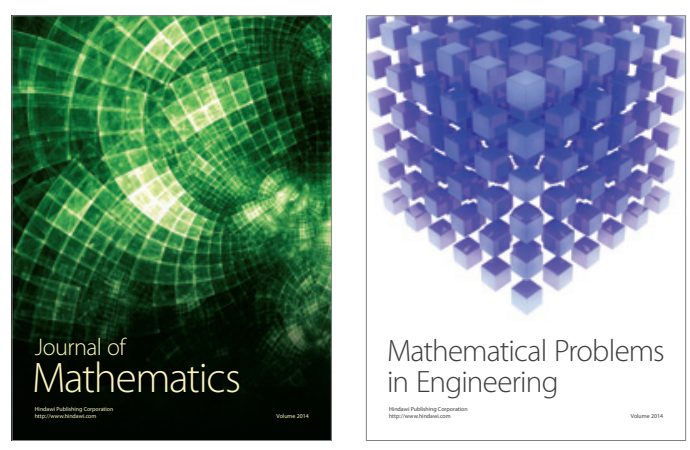

Mathematical Problems in Engineering
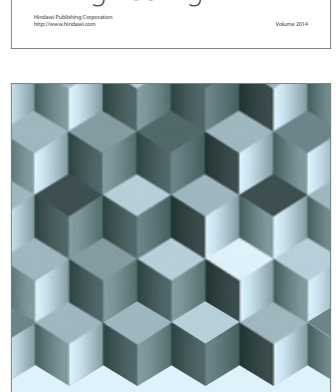

Journal of

Function Spaces
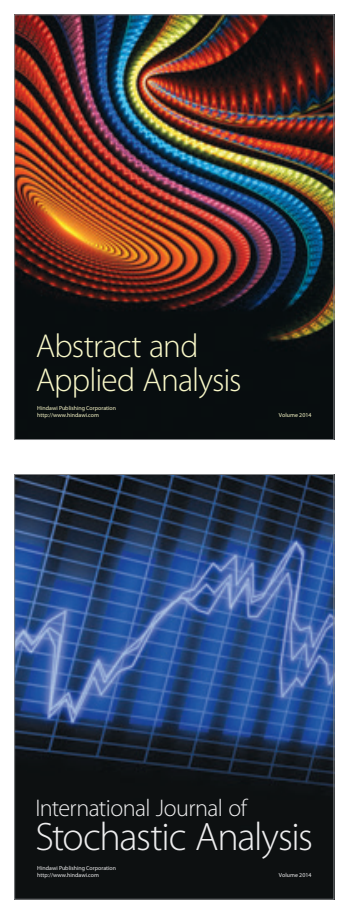

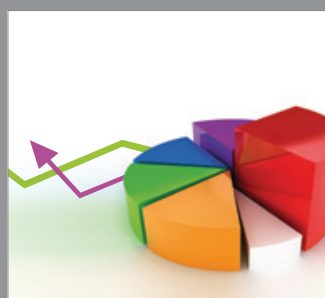

ournal of

Probability and Statistics

Promensencen
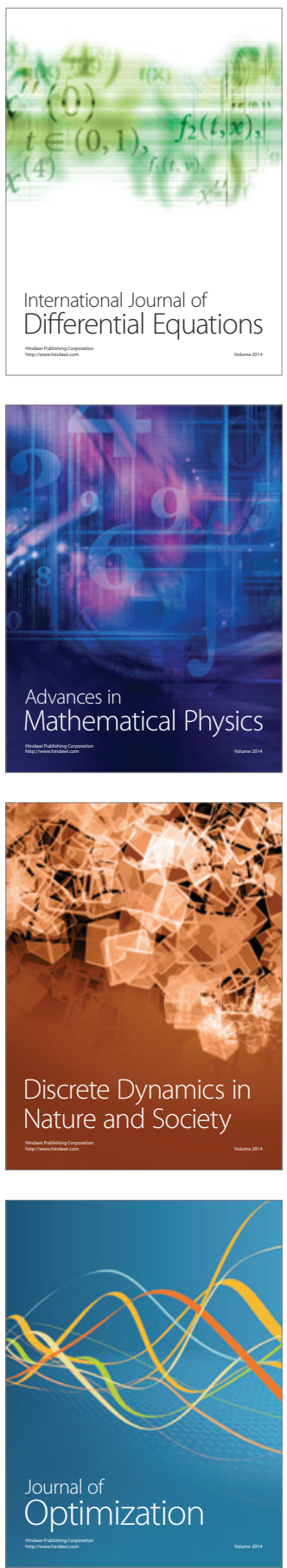